\title{
TRENDS OF PAPERS PUBLISHED FROM 2006 TO 2010 IN JOURNALS NATURE AND SCIENCE
}

\author{
Sang Chul Kim ${ }^{1}$, Seung-Lee Kim ${ }^{1}$, Jaemann Kyeong ${ }^{1}$, Jae Woo Lee $^{1}$, \\ WANGGI Lim ${ }^{1}$, AND SUng HYun JEON ${ }^{1,2}$ \\ ${ }^{1}$ Korea Astronomy and Space Science Institute, Daejeon 305-348, Republic of Korea \\ ${ }^{2}$ Department of Astronomy and Space Science, Chungnam National University, \\ Daejeon 305-764, Republic of Korea \\ E-mail: sckim@kasi.re.kr \\ (Received March 26, 2012; Accepted May 22, 2012)
}

\begin{abstract}
We present an analysis of the papers published in the journals Nature and Science in the years from 2006 to 2010. During this period, 7788 papers in total were published in the two journals. This includes 544 astronomy papers that correspond to $7.0 \%$ of the papers in 'all' research fields and $18.9 \%$ of those in the field of 'physical sciences'. The sub-fields of research of the astronomy papers are distributed, in a descending order of the number of papers, in Solar System, stellar astronomy, galaxies and the universe, the Milky Way Galaxy, and exoplanets. The observational facilities used for the studies are mainly ground-based telescopes (31.1\%), spacecrafts $(27.0 \%)$, and space telescopes $(22.8 \%$ ), while $16.0 \%$ of papers did not use any noticeable facilities and $1.7 \%$ used other facilities. Korean scientists have published 86 papers (33 in Nature and 53 in Science), which is $1.10 \%$ of all the papers $(\mathrm{N}=7788)$ in the two journals. The share of papers by Korean astronomers among the scientific papers by Koreans is $8.14 \%$, slightly higher than the contribution of astronomy papers $(7.0 \%)$ in both journals.
\end{abstract}

Key words: history and philosophy of astronomy; sociology of astronomy; astronomical data bases: miscellaneous

\section{INTRODUCTION}

While all scientific and astronomical research papers report new findings on nature and the Universe, some of them contribute greatly to the development of science and human knowledge. These achievements that have a high impact on science and mankind are often acknowledged by press releases to the public and/or prestigious prizes such as the Nobel Prize. Some representative ways to assess papers and determine which are high impact papers could be investigating highly cited papers or considering papers published in highly cited journals (Davoust \& Schmadel, 1987; Leverington, 1996; Schulman et al., 1997; Abt, 1998, 2000; Pearce, 2004;
Trimble \& Ceja, 2008; Stanek, 2008; Crabtree, 2008; Trimble, 2009; Frogel, 2010; Kim, 2011).

There have been many studies to measure the productivity and/or effectiveness of (1) facilities (e.g. telescopes) (Trimble, 1995, 1996; Benn \& Sánchez, 2001a, 2001b; Ringwald et al., 2003; Meylan, Madrid, \& Macchetto, 2004; Trimble, Zaich, \& Bosler, 2005; Grothkopf et al., 2005; Trimble \& Ceja, 2007, 2008; Trimble, 2009; Apai et al., 2010), (2) organizations (Crabtree \& Bryson, 2003), (3) countries (Sánchez \& Benn, 2004; Abt, 2010; Kamphuis \& van der Kruit, 2010), (4) scholars (Dietrich, 2008; Stanek, 2009; Kamphuis \& van der Kruit, 2010; Pimbblet, 2011), and so 
on. Ahn et al. (2008) suggested that the number of papers produced by ground-based large $(D \sim 3.6-10$ $\mathrm{m})$ optical telescopes are roughly proportional to the diameters of the primary mirrors (see also Leverington (1997)). Recently, Kim (2011) presented results of an investigation on the paper productivities of groundbased large $(D>8 \mathrm{~m})$ optical telescopes from an analysis of papers published from 2000 to 2009 .

Considering that the astronomical papers with the highest number of citations and those published in the journals Nature and Science are the outputs with the greatest impact on science and on mankind (Benn \& Sánchez, 2001b), we have investigated the papers published in the journals Nature and Science from 2006 to 2010 in this study. Specifically, we have tried to answer the following questions which people often ask: (1) how many papers are published in the journals Nature and Science, (2) what is the percentage of astronomy papers among these papers, (3) what are the distributions and portions of sub-research fields of astronomy, (4) what facilities were used for those astronomy papers and what were the percentages of their uses, and (5) how many Korean scientists and Korean astronomers contributed to those papers. Because in some countries including Korea there is insufficient capability in the society to assess the scientific competence of personnel or the qualities of research output, it is common to consider publications in highly cited journals/magazines like Nature and Science as the proxy of scientific expertise. It will be, therefore, meaningful to investigate the statistics and distribution of papers in the two representative journals. This paper is organized as follows: Section 2 describes the data utilized in this work. Section 3 presents the analysis results of the number of papers, research fields in astronomy, astronomical facilities used, and papers by Koreans. Finally, Section 4 provides summary and discussion of the results.

\section{DATA}

The academic papers investigated in this study are those contained in the two weekly journals Nature ${ }^{1}$ and Science $^{2}$ for five years from 2006 to 2010. Among the contents of the two journals, we only counted 'articles' and 'letters' in Nature and 'research articles' and 'reports' in Science in order to take into account original

\footnotetext{
${ }^{1}$ http://www.nature.com.

${ }^{2}$ http://www.sciencemag.org.
}

studies (cf. Isaac Newton Group webpage ${ }^{3}$ ).

In this paper, we have used the term 'astronomy' to include both astronomy and astrophysics.

\section{RESULTS}

\subsection{Number of Papers}

Table 1 shows the basic statistics of the papers in the journals Nature and Science from 2006 to 2010. During this period, 4004 papers were published in $N a$ ture, while 3784 papers were published in Science, with yearly mean numbers of 800 and 757 , and weekly (i.e., per issue) mean numbers of 15.6 and 14.8, respectively.

For the journal Nature, we have used the webpage of the Japanese table of contents, which shows a detailed field classification for each article. Using these classifications, we distributed each research field into two areas of life sciences and physical sciences, of which items are shown in the footnotes of Table 1. The second column of Table 1 shows the yearly number of papers in each of these two main categories for the journal $\mathrm{Na}$ ture.

The subject index of the journal Science's webpage gives three main classifications; life sciences, physical sciences, and other subjects. Astronomy is included in the physical sciences; 'other subjects' include education, economics, sociology, policy/research ethics, etc. The numbers of papers in each of these categories for each year are shown in the fourth column of Table 1.

For the period of 2006 to 2010, there were 319 and 225 astronomy papers published in the journals $\mathrm{Na}$ ture and Science, with yearly mean values of 64 and 45 , respectively. The astronomy papers in Nature accounted for $8.0 \%$ and $24.1 \%$ of 'all' and 'physical sciences' papers, while those of Science accounted for $5.9 \%$ and $14.4 \%$, respectively. These $544(=319+225)$ papers in astronomy for the journals Nature and Science comprise a total of $7.0 \%$ of the papers for 'all' research fields $(\mathrm{N}=7788=4004+3784)$ and $18.9 \%$ of the papers for the fields of 'physical sciences' (N $=2885=1321+1564)$.

If we simply compare the fraction of astronomy papers among all science papers with the fraction of funds given to astronomy field among all research fields, the portion of astronomy papers among all science papers is greater than that of the fund given to astronomy among all the research related budgets. For example, in the

\footnotetext{
${ }^{3}$ http://www.ing.iac.es/PR/natsci.html.
} 


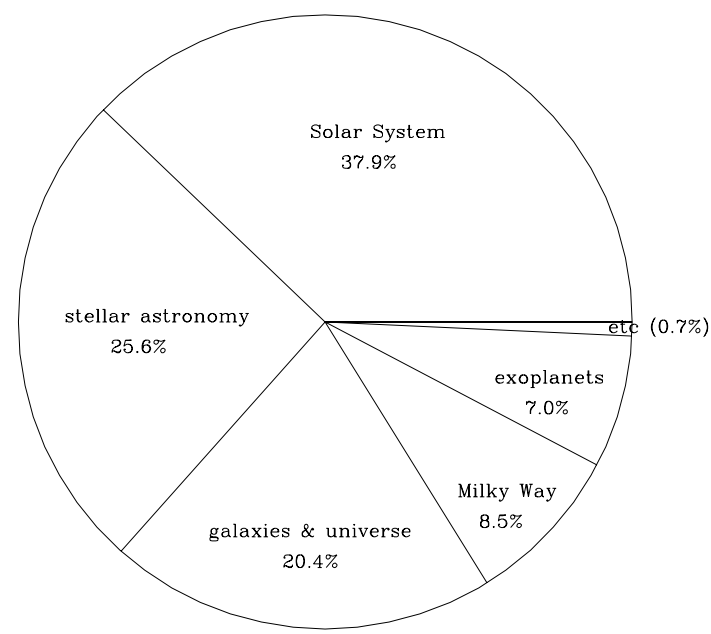

Fig. 1.- The distribution of subjects for papers published in the journals Nature and Science. 'stellar astronomy' includes stars, supernovae, novae, star formation, and the Sun; 'galaxies \& universe' includes external galaxies, active galactic nuclei, gamma-ray bursts, galaxy clusters, large scale structure, and cosmology; 'Milky Way' includes our Galaxy, star clusters, and interstellar matter; 'etc.' includes cosmic rays and instrumentation. This shows only the general trend and the exact order could change, depending on the classification criteria of each item.

case of the United States (we take the U.S. case as an example because it is not easy to get funding information for other countries), funds approved and disbursed by the National Science Foundation for the field of astronomy in 2011 were only $4.3 \%$ (236.6 million USD) of the research and related activities (R\&RA) fund of 5.56 billion $\mathrm{USD}^{4}$. All the more interesting is to note that astronomy papers make up almost one fifth of the physical science papers, which include all the natural sciences and engineering fields. This implies that astronomy has a greater impact on science and mankind and generates great interest from the general public.

\subsection{Sub-fields in Astronomy}

Table 2 shows the distribution of papers in the subfields of astronomy for the journals Nature and Science.

\footnotetext{
${ }^{4}$ http://www.nsf.gov.
}

The classification criterion has been set up by the authors and modified by comparing the results from the two journals. Figure 1 shows the general distribution of the sub-fields. The data in this Figure shows that the order, sorted by number of papers, is Solar System, stellar astronomy, galaxies and the universe, the Milky Way Galaxy, and exoplanets.

'Solar System' is the most studied sub-field in the two journals, with a percentage of $37.9 \%$, followed by 'Stars' (11.4\%), 'External Galaxies' (10.5\%), 'Supernovae and Novae' (7.2\%), 'Exoplanets' (7.0\%), and so on (Table 2). 'Solar System' and 'Stars' comprise half of astronomy papers (see the sixth column of Table 2), while the five fields (Solar System, Stars, External Galaxies, Supernovae and Novae, and Exoplanets) make up three quarters.

Reasons why the field of 'Solar System' takes the largest portion of astronomy papers could be the following. The first reason could be the launches of several spacecrafts/satellites, which bring us much closer and more detailed views/information on Solar System objects. This is shown in the following subsection, in Table 3 and in Figure 2. As can be seen in Figure 2 (a), the papers that used spacecrafts comprised $27.0 \%$ (147/544) of all the astronomy papers; and spacecrafts were used in $32.2 \%(147 / 457)$ of astronomy papers (excluding papers of 'no facility used'). Another reason might be the great interest of both scientists and the public in the neighborhood of our home planet, which extends from the Earth and Moon to Mercury, Venus, and Mars, and on to the far side of the Solar System, as well as to asteroids and comets.

\subsection{Facilities for Astronomy Papers}

Table 3 and Figure 2 show the statistics of the observational facilities used in the astronomy papers under consideration. When multiple facilities are used in a certain paper, we either (1) focused on the main facility which is presumed to have provided the most critical data for the research (e.g., taking the Very Large Telescope instead of the Keck Telescope in Gal-Yam et al. (2009)'s paper), or (2) took the larger (more expensive) facility over the smaller (cheaper) ones when they were used with similar importance (e.g., taking the 8.2 $\mathrm{m}$ Subaru telescope instead of the $2.2 \mathrm{~m} / 3.5 \mathrm{~m}$ telescopes at the Calar Alto Astronomical Observatory, in Krause et al. (2008)). Although these selections can leave some ambiguities in certain cases, we assume that 
Table 1: Number of Papers for Research Fields Published in the Nature and Science Journals

\begin{tabular}{|c|c|c|c|c|}
\hline Year & Nature & & Science & \\
\hline (1) & $\begin{array}{l}\mathrm{N}(\text { all })^{\mathrm{a}} \\
(2)\end{array}$ & $\begin{array}{l}\mathrm{N}(\text { astronomy })^{\mathrm{b}} \\
(3)\end{array}$ & $\begin{array}{l}\mathrm{N}(\text { all })^{\mathrm{c}} \\
(4)\end{array}$ & $\begin{array}{l}\mathrm{N}(\text { astronomy })^{\mathrm{b}} \\
(5)\end{array}$ \\
\hline 2006 & $822(=535+287)$ & $82(10.0 \%, 28.6 \%)$ & $758(=434+313+11)$ & $41(5.4 \%, 13.1 \%)$ \\
\hline 2007 & $762(=512+250)$ & $50(6.6 \%, 20.0 \%)$ & $743(=431+303+9)$ & $34(4.6 \%, 11.2 \%)$ \\
\hline 2008 & $823(=555+268)$ & $65(7.9 \%, 24.3 \%)$ & $748(=432+308+8)$ & $46(6.1 \%, 14.9 \%)$ \\
\hline 2009 & $783(=515+268)$ & $62(7.9 \%, 23.1 \%)$ & $774(=443+323+8)$ & $48(6.2 \%, 14.9 \%)$ \\
\hline 2010 & $814(=566+248)$ & $60(7.4 \%, 24.2 \%)$ & $761(=437+317+7)$ & $56(7.4 \%, 17.7 \%)$ \\
\hline Sum & $\begin{array}{l}4004(=2683+1321) \\
100(=67.0+33.0) \%\end{array}$ & $319(8.0 \%, 24.1 \%)$ & $\begin{array}{l}3784(=2177+1564+43) \\
100(=57.5+41.3+1.1) \%\end{array}$ & $225(5.9 \%, 14.4 \%)$ \\
\hline
\end{tabular}

a Number of papers for all research fields (= life sciences + physical sciences), where astronomy is included in the latter. Life sciences mean archaeology, structural biology, agronomy, nano-technology, brain, immunology, microbiology, virus, ontogeny, pathology, taxonomy, physiology, biophysics, ecology, biochemistry, cytology, sense, plant, neurology, psychology, cancer, pharmacology, linguistics, epidemiology, genetics, cognizance, medicine, tumor, evolution, and cooperative action and physical sciences mean engineering, nano-technology [different from that in the life science], mathematics, universe, meteorology, physics, physical chemistry, optics, quantum, materials, earth, statistics, microscopy, ocean, visualization, chemistry, chemical engineering, and environment.

b Number of papers in astronomy. Values in parentheses are percentage among 'all' papers (for the former) and percentage among 'physical sciences' papers (for the latter).

c Number of papers for all research fields (= life sciences + physical sciences + Etc.), where 'Etc.' includes, e.g., education, economics, sociology, and policy/research ethics (astronomy is included in the 'physical sciences').

they do not significantly affect the distribution shown in Figure 2.

While $16.0 \%$ of papers did not use any noticeable facilities for their studies (e.g., theory, simulation), large (D $>8 \mathrm{~m}$ ) optical telescopes were dominantly used for the studies of $10.5 \%$ papers (combining Nature and Science). They are currently the largest facilities in optical wavebands. Table 3 shows that the next most heavily-used facilities were spacecrafts to Saturn and Mars (14.9\%) and space telescopes in the gamma-ray, optical, and infrared wavebands (15.8\%). Figure 2 (a) shows that the percentages of papers that used ground-based telescopes, spacecrafts, and space telescopes were $31.1 \%, 27.0 \%$, and $22.8 \%$, respectively, for all astronomy papers. Optical and radio \& sub-mm telescopes make up $69.2 \%$ and $23.7 \%$, respectively, of all the ground-based telescopes (Figure $2(\mathrm{~b})$ ). Figure 2 (c) shows that space telescopes of gamma-ray, optical, infrared, X-ray, and ultraviolet wavebands take $24.2 \%$, $23.4 \%, 21.8 \%, 16.1 \%$, and $3.2 \%$, respectively.

Among the 105 papers that used ground-based optical telescopes, as shown in Figure 2 (d), the largest telescopes of $\mathrm{D}>8 \mathrm{~m}$ comprise $54.3 \%(\mathrm{~N}=57)$, while those of $3.0 \leq \mathrm{D} \leq 4.2 \mathrm{~m}$ take $21.0 \%(\mathrm{~N}=22)$, those of $\mathrm{D}<3.0 \mathrm{~m}$ take $19.0 \%(\mathrm{~N}=20)$ and those of 5.0 $\leq \mathrm{D} \leq 6.5 \mathrm{~m}$ take $5.7 \%(\mathrm{~N}=6)$. The possible reason why ground-based optical telescopes of diameters between $5.0 \mathrm{~m}$ and $6.5 \mathrm{~m}$ take a lesser percentage of papers (1.1\% among all the facilities in Table 3$)$ than those of diameters between $3.0 \mathrm{~m}$ and $4.2 \mathrm{~m}(4.0 \%)$ or even those of diameters smaller than $3.0 \mathrm{~m}(3.7 \%)$ (Figure $2(\mathrm{~d})$ ) could be the lower $(\lesssim 1 / 3)$ number of telescopes for ground-based optical telescopes of diameters between $5.0 \mathrm{~m}$ and $6.5 \mathrm{~m}$. As can be seen in Table 3, among the ground-based optical telescopes, it is remarkable that the Sloan Digital Sky Survey (SDSS) project, operated with only one $2.5 \mathrm{~m}$ telescope, stands out with $2.2 \%$ of papers. This might result from a large survey program covering a quarter of the sky and the creation of 3-dimensional maps containing more than 930,000 galaxies and more than 120,000 quasars via both photometry and spectroscopy (Abazajian et al., 2009). Although the large fraction of ground-based optical telescopes of D $>8 \mathrm{~m}$ (Figure $2(\mathrm{~d})$ ) could be somewhat biased, if at all, by the second criterion ex- 
Table 2: Sub-field Distribution of Astronomical Papers in the Nature and Science Journals from 2006 to $2010^{\mathrm{a}}$

\begin{tabular}{|c|c|c|c|c|c|}
\hline Field & $\mathrm{N}($ Nature $)$ & $\mathrm{N}($ Science $)$ & $\mathrm{N}(\mathrm{Sum})$ & Percentage [\%] & $\begin{array}{l}\text { Accumulated } \\
\text { Percentage }[\%\end{array}$ \\
\hline$(1)$ & $(2)$ & $(3)$ & $(4)$ & $(5)$ & $(6)$ \\
\hline Solar System & 107 & 99 & 206 & 37.9 & 37.9 \\
\hline Stars & 32 & 30 & 62 & 11.4 & 49.3 \\
\hline External Galaxies & 45 & 12 & 57 & 10.5 & 59.7 \\
\hline Supernovae and Novae & 23 & 16 & 39 & 7.2 & 66.9 \\
\hline Exoplanets & 28 & 10 & 38 & 7.0 & 73.9 \\
\hline Formation of Stars and the Solar System & 13 & 14 & 27 & 5.0 & 78.9 \\
\hline Interstellar Matter (including Supernova Remnants) & 11 & 12 & 23 & 4.2 & 83.1 \\
\hline Gamma Ray Bursts & 17 & 5 & 22 & 4.0 & 87.1 \\
\hline Cosmology & 10 & 4 & 14 & 2.6 & 89.7 \\
\hline Milky Way Galaxy & 8 & 4 & 12 & 2.2 & 91.9 \\
\hline Star Clusters & 7 & 4 & 11 & 2.0 & 93.9 \\
\hline Sun & 6 & 5 & 11 & 2.0 & 96.0 \\
\hline Active Galactic Nuclei & 7 & 3 & 10 & 1.8 & 97.8 \\
\hline Galaxy Clusters and Large Scale Structure & 3 & 5 & 8 & 1.5 & 99.3 \\
\hline Cosmic Ray & 1 & 2 & 3 & 0.6 & 99.9 \\
\hline Instrumentation & 1 & 0 & 1 & 0.2 & 100 \\
\hline Sum & 319 & 225 & 544 & 100 & - \\
\hline
\end{tabular}

${ }^{\text {a }}$ On the order of percentage.

plained in the first paragraph of this subsection, it is still valuable to note that state-of-the-art facilities and big science (e.g. space telescopes, ground-based optical telescopes of D $>8 \mathrm{~m}$ ) and dedicated facilities (e.g. SDSS, Cassini, Voyager, CoRoT) might be one of the critical factors to create high impact papers.

\subsection{Papers by Koreans}

For the papers published in Nature and Science from 2006 to 2010, we have probed the papers with Korean scientists in the author lists and show the results in Table 4. We extracted papers with authors of Korean names and Korean affiliations.

Table 4 shows that 86 papers (33 for Nature and 53 for Science) have Koreans as the authors, and among them seven papers (two for Nature and five for Science) are astronomy papers written by Korean astronomers. In total, Korean authors contributed $1.10 \%$ of the total 7788 papers published in the two journals. Astronomy papers by Korean authors $(\mathrm{N}=7)$ make up $8.14 \%$ of the 86 papers by Korean scientists. While astronomy papers comprise $7.0 \%$ of the papers of 'all' research fields in the two journals Nature and Science, as can be seen in Section 3.1, this contribution of Korean astronomers to the Korean sciences (at least in the statistics of the two journals for the given period) shows a slightly higher percentage than the world normal. If we take only the first author and corresponding author papers, the rate even increases to $11.1 \%$ (5/45).

Table 5 shows the number distribution of papers written by Korean scientists in the fields of physics, chemistry (including biochemistry), biology \& life science, earth sciences \& astronomy, and engineering (including materials science). Although biology \& life science is the field of most abundance and earth sciences \& astronomy is the field with least number of papers, the fraction of papers in biology \& life science in Korea $(29.1 \%)$ is much lower than the fraction of this field in the world (Table $1 ; 4860 / 7788=62.4 \%$ ). It is also worthwhile to note that astronomy papers make up two thirds $(7 / 11)$ of the papers in the field of earth sciences \& astronomy.

Table 6 provides a detailed bibliography of the seven Nature and Science papers written by Korean 
Table 3: Facilities Used in the Papers of Nature and Science Journals from 2006 to 2010

\begin{tabular}{|c|c|c|c|c|}
\hline Facility & Nature & Science & Sum (\%) & $\begin{array}{c}\text { Accumulated } \\
\text { Percentage }[\%]^{\mathrm{a}} \\
(5)\end{array}$ \\
\hline no facility used & 58 & 29 & $87(16.0)$ & - \\
\hline ground-based telescope, optical (D > $8 \mathrm{~m}$ ) & 39 & 18 & $57(10.5)$ & 10.5 \\
\hline spacecraft - Cassini & 28 & 18 & $46(8.5)$ & 18.9 \\
\hline spacecrafts to Mars & 12 & 23 & $35(6.4)$ & 25.4 \\
\hline space telescope, gamma-ray & 15 & 15 & $30(5.5)$ & 30.9 \\
\hline space telescope, optical & 24 & 5 & $29(5.3)$ & 36.2 \\
\hline space telescope, infrared & 18 & 9 & $27(5.0)$ & 41.2 \\
\hline radio telescope & 13 & 11 & $24(4.4)$ & 45.6 \\
\hline ground-based telescope, optical $(3.0 \leq \mathrm{D} \leq 4.2 \mathrm{~m})$ & 19 & 3 & $22(4.0)$ & 49.6 \\
\hline spacecrafts to Moon & 2 & 20 & $22(4.0)$ & 54.0 \\
\hline ground-based telescope, optical (D < $3.0 \mathrm{~m})$ & 16 & 4 & $20(3.7)$ & 57.3 \\
\hline space telescope, X-ray & 13 & 7 & $20(3.7)$ & 61.0 \\
\hline SDSS & 8 & 4 & $12(2.2)$ & 63.2 \\
\hline VLA, VLBA, VLBI & 5 & 7 & $12(2.2)$ & 65.4 \\
\hline spacecraft - Others & 4 & 8 & $12(2.2)$ & 67.6 \\
\hline spacecraft - Voyager & 8 & 2 & $10(1.8)$ & 69.5 \\
\hline ground-based telescope, gamma-ray & 3 & 5 & $8(1.5)$ & 70.9 \\
\hline supercomputer & 5 & 3 & $8(1.5)$ & 72.4 \\
\hline spacecrafts to Mercury & 0 & 7 & $7(1.3)$ & 73.7 \\
\hline spacecrafts to Earth & 1 & 6 & $7(1.3)$ & 75.0 \\
\hline ground-based telescope, optical $(5.0 \leq \mathrm{D} \leq 6.5 \mathrm{~m})$ & 5 & 1 & $6(1.1)$ & 76.1 \\
\hline CoRoT satellite & 4 & 2 & $6(1.1)$ & 77.2 \\
\hline Lunar sample/meteorite & 5 & 1 & $6(1.1)$ & 78.3 \\
\hline submm telescope & 2 & 2 & $4(0.7)$ & 79.0 \\
\hline space telescope, ultraviolet & 3 & 1 & $4(0.7)$ & 79.8 \\
\hline space telescope, Sun observing & 1 & 2 & $3(0.6)$ & 80.3 \\
\hline balloon & 2 & 0 & $2(0.4)$ & 80.7 \\
\hline ground-based telescope, Sun observing & 0 & 2 & $2(0.4)$ & 81.1 \\
\hline ground-based telescope, cosmic ray & 0 & 2 & $2(0.4)$ & 81.4 \\
\hline space telescope - Kepler & 0 & 2 & $2(0.4)$ & 81.8 \\
\hline spacecrafts to Venus & 1 & 1 & $2(0.4)$ & 82.2 \\
\hline space telescope - WMAP & 0 & 1 & $1(0.2)$ & 82.3 \\
\hline etc. $^{\mathrm{b}}$ & 5 & 4 & $9(1.7)$ & 84.0 \\
\hline Total & 319 & 225 & $544(100)$ & - \\
\hline
\end{tabular}

a Excludes the first row of 'no facility used' (16.0\%). Accumulation starts from the second item (optical telescope, D > 8 $\mathrm{m})$ and the final sum becomes $84.0 \%$.

b Includes virtual observatory, Center for High Angular Resolution Astronomy (CHARA interferometer), Navy Prototype Optical Interferometer (NPOI), dark matter search detector array, Laser Interferometer Gravitational-wave Observatory (LIGO), composition analyzer, velocimeter, and magnetometer. 


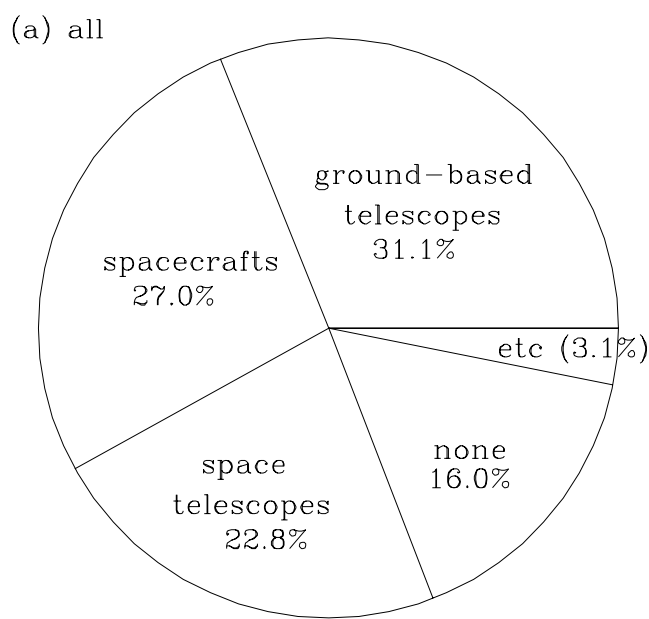

(c) space telescopes

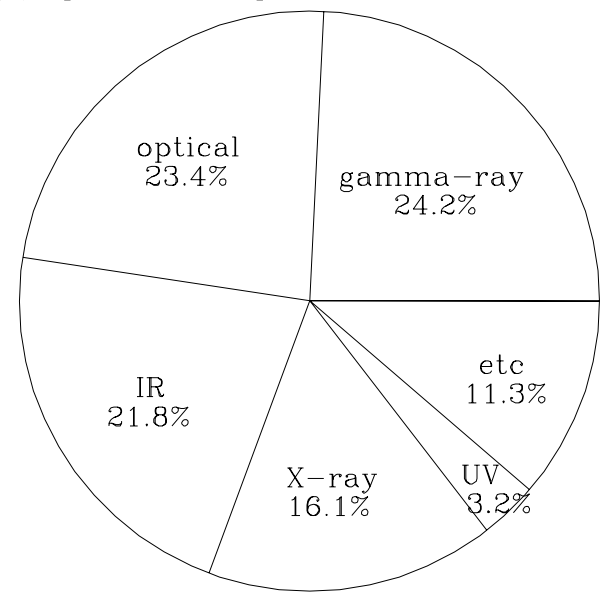

(b) ground-based telescopes

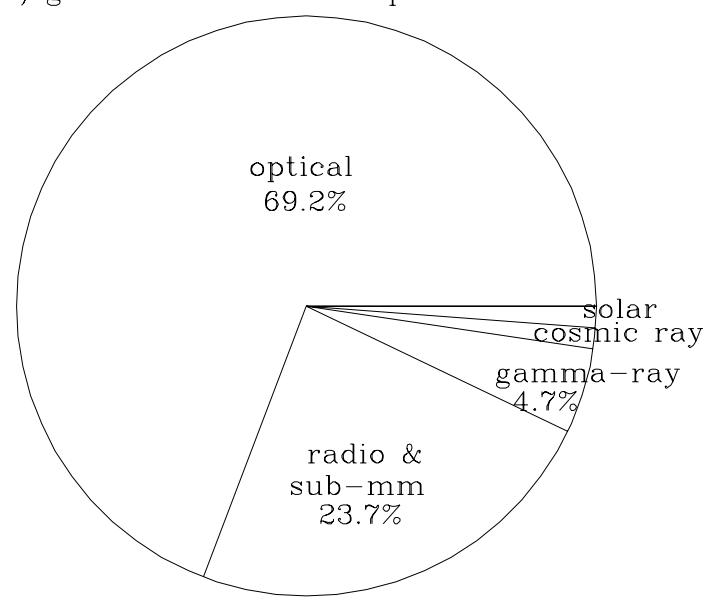

(d) ground-based optical telescopes

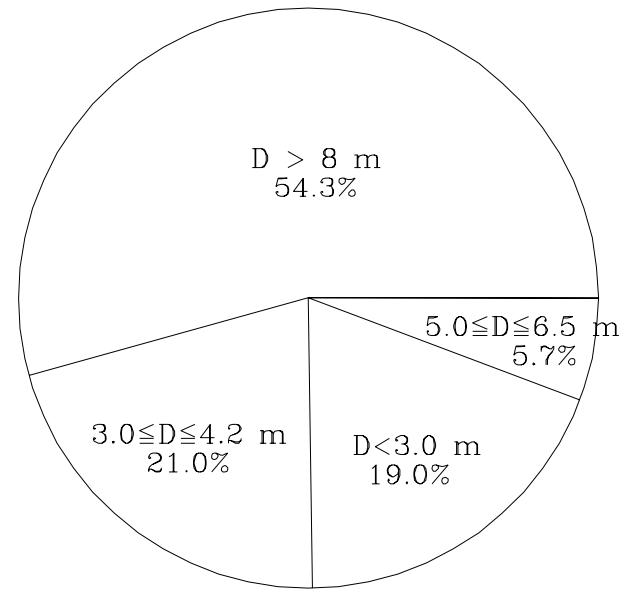

Fig. 2. - Pie charts for the facilities used in astronomy papers published in the journals Nature or Science from 2006 to 2010. (a) Facilities for all 544 astronomy papers. Spacecrafts include Lunar sample/meteorite; space telescopes include balloon and CoRoT satellites; 'etc.' includes supercomputer and the last item in Table 3. (b) Sub-distribution of ground-based telescopes for all wavelength ranges. 'Optical' includes the SDSS telescope, and 'radio \& sub-mm' includes VLA, VBLA, and VLBI telescopes. (c) Sub-distribution of space telescopes, where 'etc.' contains solar telescopes, Kepler, WMAP, balloons, and the CoRoT satellite. (d) Sub-distribution of ground-based optical telescopes. 
astronomers from 2006 and 2010, of which five papers are with first/corresponding authors and the remaining two are with co-authors. Among the seven papers, two (Yoon, Yi, \& Lee, 2006; Ryu et al., 2008) used no observational facilities; two papers that used GALEX ${ }^{5}$ as the main facility (Schawinski et al., 2006, 2008) actually used multiple facilities; one paper (Lee, Park, \& Hwang, 2010) used the SDSS data; and the remaining two papers (Lee et al., 2009; Gaudi et al., 2008) used small optical telescopes of $1 \mathrm{~m}$ class. This status of facilities used by Korean astronomers reflects well the current situation of facilities for the Korean astronomical community, and shows (1) participation in one space project (GALEX), (2) use of small ground-based optical telescopes (CTIO $1.0 \mathrm{~m}$ and Mt. Lemmon 1.0 m telescopes), (3) use of public archive data (SDSS), and (4) studies without any noticeable facilities. Since the tools that we use to look at the Universe are essential in astronomical studies, as can be seen in the Table 3, construction of or participation in more facilities/projects will bring a greater number of Nature and Science papers in the future.

While the sub-fields of the seven Nature and Science papers published by Korean astronomers are diverse, it is interesting that three $(43 \%)$ out of seven papers are on 'star clusters'. It is true, in general, that scientists with more and better facilities produce more and better papers. Nevertheless, considering the fact that these three papers did not use any of the current largest (or most expensive) facilities, this statistic shows that excellence in scholarship is another indispensable element in its own way.

\section{SUMMARY AND DISCUSSION}

We have examined the distribution and statistics of 'articles' and 'letters' in the journal Nature and 'research articles' and 'reports' in the journal Science published from 2006 to 2010. The 4004 Nature papers are composed of 2683 life science papers and 1321 physical science papers, among which the latter group contains 319 (8.0\% among 4004) astronomy papers. The 3784 Science papers are made up of 2177 life science papers, 1564 physical science papers, and 43 papers in other fields, where 225 (5.9\% among 3784) astronomy papers are included in the physical science papers. In total, astronomy papers comprise $7.0 \%$ of the papers for 'all' research fields and $18.9 \%$ of the papers for the fields of

\footnotetext{
${ }^{5}$ Galaxy Evolution Explorer satellite.
}

'physical sciences' in the two journals.

The sub-fields of study for these astronomy papers are as follows: 'Solar System' (37.9\%) and 'Stars' $(11.4 \%)$ comprise half of the astronomy papers, while the five fields of Solar System, Stars, External Galaxies, Supernovae and Novae, and Exoplanets make up three quarters.

While $16 \%$ of the astronomy papers did not use any noticeable facilities for their research, spacecrafts, space telescopes, and ground-based telescopes were used for $27.0 \%, 22.8 \%$, and $31.1 \%$, respectively. Such spacecrafts, which explore in detail objects in the Solar System, might have been an important factor in increasing the large number of the research papers in this field. Space telescopes are mainly those in the gammaray $(24.2 \%)$, optical $(23.4 \%)$, infrared $(21.8 \%)$, and $\mathrm{X}$ ray $(16.1 \%)$ wavebands; ground-based telescopes are largely optical $(69.2 \%)$ and radio $(23.7 \%)$ telescopes. The largest $(\mathrm{D}>8 \mathrm{~m}$ ) ground-based optical telescopes produced the greatest number of Nature and Science papers $(57 / 105,54.3 \%)$ among all ground-based telescopes. While this value could be affected somewhat, if not much, by one of the facility-selection criteria in Section 3.3 (taking the larger facility as the primary facility for the paper when one large and one small facilities are used in equal amounts), it seems that the order of importance among the ground-based optical telescopes would not change.

From the 4004 Nature and 3784 Science papers, we have extracted 86 papers by Korean authors with Korean affiliations. Among these 86 papers, seven astronomy papers (two in Nature and five in Science) are included, making up $8.14 \%$ of the 86 Korean papers. While 'astronomy' papers comprise $7.0 \%$ of the papers for 'all' the research fields of the journals Nature and Science, Korean astronomers appear to contribute slightly more $(8.14 \%)$ to all Korean papers in these two journals.

We anticipate that these results might be used, at least, for establishing criteria to assess leading research groups (especially for astronomy fields), and estimating future production of Nature and Science papers.

\section{ACKNOWLEDGEMENTS}

We would like to thank the anonymous referees for the very helpful comments and clarifications that helped to improve the manuscript. We would also like 
Table 4: Papers Written by Korean Scientists ${ }^{\text {a }}$ from 2006 to 2010

\begin{tabular}{|c|c|c|c|c|c|c|}
\hline \multirow[t]{2}{*}{ Journal } & \multicolumn{3}{|c|}{ All Fields ${ }^{\mathrm{b}}$} & \multicolumn{3}{|c|}{ Astronomy } \\
\hline & $\mathrm{N}$ & $\left(=\right.$ First Author ${ }^{\mathrm{c}}+$ & Others) & $\mathrm{N}$ & $\left(=\right.$ First Author ${ }^{\mathrm{c}}+$ & Others) \\
\hline Nature & $\begin{array}{c}33 \\
0.82 \%\end{array}$ & $\begin{array}{c}\quad=17+ \\
\left(=0.42 \%^{\mathrm{d}}+\right.\end{array}$ & $\begin{array}{c}16) \\
\left.0.40 \%{ }^{\mathrm{d}}\right)\end{array}$ & $\begin{array}{c}2 \\
6.06 \%\end{array}$ & $(=2+$ & 0) \\
\hline Science & $\begin{array}{c}53 \\
1.40 \%\end{array}$ & $\begin{array}{c}(=28+ \\
\left(=0.74 \%^{\mathrm{f}}+\right.\end{array}$ & $\begin{array}{c}25) \\
\left.0.66 \%^{f}\right)\end{array}$ & $\begin{array}{c}5 \\
9.43 \%^{\mathrm{g}}\end{array}$ & $(=3+$ & 2) \\
\hline Sum & $\begin{array}{c}86 \\
1.10 \%^{\mathrm{h}}\end{array}$ & $\begin{array}{c}(=45+ \\
\left(=0.58 \%^{\mathrm{h}}+\right.\end{array}$ & $\begin{array}{c}41) \\
\left.0.53 \%^{\mathrm{h}}\right)\end{array}$ & $\begin{array}{c}7 \\
8.14 \%^{\mathrm{i}}\end{array}$ & $(=5+$ & 2) \\
\hline
\end{tabular}

${ }^{\text {a }}$ Korean names and Korean affiliations.

b Including astronomy.

${ }^{c}$ Including corresponding author.

d Percentage among the 4004 Nature papers from 2006 to 2010.

e Percentage among the 33 Nature papers by Korean scientists from 2006 to 2010.

${ }^{\mathrm{f}}$ Percentage among the 3784 Science papers from 2006 to 2010.

g Percentage among the 53 Science papers by Korean scientists from 2006 to 2010.

h Percentage among the 7788 Nature and Science papers from 2006 to 2010.

i Percentage among the 86 papers by Korean scientists from 2006 to 2010.

Table 5: Number of Papers Written by Koreans ${ }^{\mathrm{a}}$ in Each Field

\begin{tabular}{clllll}
\hline \hline & Nature & \multicolumn{3}{c}{ Science } & Sum(\%) \\
Field & First Author $^{\mathrm{b}}$ & Others & First Author $^{\mathrm{b}}$ & Others \\
\hline Physics & 6 & 6 & 3 & 7 & $22(25.6 \%)$ \\
Chemistry & 4 & 1 & 4 & 5 & $14(16.3 \%)$ \\
Biology \& Life Science & 1 & 7 & 12 & 5 & $25(29.1 \%)$ \\
Earth Science \& Astronomy & $3(2)^{\mathrm{c}}$ & 0 & $4(3)^{\mathrm{c}}$ & $4(2)^{\mathrm{c}}$ & $11(12.8 \%)$ \\
Engineering & 3 & 2 & 5 & 4 & $14(16.3 \%)$ \\
\hline Sum & 17 & 16 & 28 & 25 & $86(100 \%)$ \\
\hline
\end{tabular}

\footnotetext{
${ }^{\text {a }}$ Korean names and Korean affiliations.

${ }^{\mathrm{b}}$ Including corresponding author.

c The number in parentheses is that for astronomy.
} 
Table 6: Bibliography of Papers Written by Korean Astronomers from 2006 to $2010^{\mathrm{a}}$

\begin{tabular}{|c|c|c|}
\hline \multirow[t]{5}{*}{$1^{\mathrm{b}}$} & Authors & $\begin{array}{l}\text { Schawinski, Kevin; Khochfar, Sadegh; Kaviraj, Sugata; Yi, Sukyoung K.; } 15 \text { coauthors ; } \\
\text { Lee, Young-Wook; and } 4 \text { coauthors }\end{array}$ \\
\hline & Journal & 2006, Nature, 442,888 \\
\hline & Title & Suppression of star formation in early-type galaxies by feedback from supermassive black holes \\
\hline & Facilities & GALEX, SDSS \\
\hline & Subject & External Galaxies \\
\hline \multirow[t]{5}{*}{$2^{\mathrm{c}}$} & Authors & Lee, Jae-Woo; Kang, Young-Woon; Lee, Jina; Lee, Young-Wook \\
\hline & Journal & 2009, Nature, 462, 480 \\
\hline & Title & Enrichment by supernovae in globular clusters with multiple populations \\
\hline & Facilities & CTIO $1.0 \mathrm{~m}$ \\
\hline & Subject & Star Clusters \\
\hline \multirow[t]{5}{*}{$3^{\mathrm{c}}$} & Authors & Yoon, Suk-Jin; Yi, Sukyoung Ken; Lee, Young-Wook \\
\hline & Journal & 2006, Science, 311,1129 \\
\hline & Title & Explaining the Color Distributions of Globular Cluster Systems in Elliptical Galaxies \\
\hline & Facilities & Models \\
\hline & Subject & Star Clusters \\
\hline \multirow[t]{5}{*}{$4^{\mathrm{c}}$} & Authors & Ryu, Dongsu; Kang, Hyesung; Cho, Jungyeon; Das, Santabrata \\
\hline & Journal & 2008, Science, 320,909 \\
\hline & Title & Turbulence and Magnetic Fields in the Large-Scale Structure of the Universe \\
\hline & Facilities & Simulations \\
\hline & Subject & Large-Scale structure \\
\hline \multirow[t]{5}{*}{$5^{\mathrm{c}}$} & Authors & Lee, Myung Gyoon; Park, Hong Soo; Hwang, Ho Seong \\
\hline & Journal & 2010, Science, 328,334 \\
\hline & Title & Detection of a Large-Scale Structure of Intracluster Globular Clusters in the Virgo Cluster \\
\hline & Facilities & SDSS \\
\hline & Subject & Star Clusters \\
\hline \multirow[t]{5}{*}{$6^{\mathrm{d}}$} & Authors & $\begin{array}{l}\text { Gaudi, B. S.; } 19 \text { coauthors ; Han, C.; Kaspi, S.; Lee, C.-U.; } 3 \text { coauthors ; Park, B.-G.; } \\
\text { and } 47 \text { coauthors }\end{array}$ \\
\hline & Journal & 2008, Science, 319,927 \\
\hline & Title & Discovery of a Jupiter/Saturn Analog with Gravitational Microlensing \\
\hline & Facilities & LOAO and many telescopes \\
\hline & Subject & Exoplanets \\
\hline \multirow[t]{5}{*}{$7^{\mathrm{d}}$} & Authors & Schawinski, Kevin; 22 coauthors; Yi, Sukyoung K. \\
\hline & Journal & 2008, Science, 321,223 \\
\hline & Title & Supernova Shock Breakout from a Red Supergiant \\
\hline & Facilities & GALEX, CFHT, VLT, Gemini, HST \\
\hline & Subject & Supernovae \\
\hline
\end{tabular}

a Names of non-Korean authors in the middle of author list are substituted with number of coauthors.

b Paper with Korean corresponding author (Yi, Sukyoung K.).

${ }^{\mathrm{c}}$ First author papers.

d Coauthor papers. 
to thank Dr. Byeong-Gon Park for helping us with the NSF related information.

\section{REFERENCES}

Abazajian, K. N., et al., 2009, The Seventh Data Release of the Sloan Digital Sky Survey, ApJS, 182, 543

Abt, H. A., 1998, Is the Astronomical Literature Still Expanding Exponentially?, PASP, 110, 210

Abt, H. A., 2000, Astronomical Publications in the Near Future, PASP, 112, 1417

Abt, H. A., 2010, Have We Reached a Maximum Astronomical Research Output?, PASP, 122, 955

Ahn, S.-H., Park, B.-G., Kim, Y.-S., Chun, M.-Y., Kim, H.-I., Sung, H.-I., Lee, D.-W., \& Kim, S. C., 2008, Estimated Productivity of the Giant Magellan Telescope, PKAS, 23, 123 (Erratum: 2010, PKAS, 25, 51)

Apai, D., Lagerstrom, J., Reid, I. N., Levay, K. L., Fraser, E., Nota, A., \& Henneken, E., 2010, Lessons from a High-Impact Observatory: The Hubble Space Telescope's Science Productivity between 1998 and 2008, PASP, 122, 808

Benn, C. R. \& Sánchez, S. F., 2001a, Scientific Impact of Large Telescopes, PASP, 113, 385

Benn, C. \& Sánchez, S., 2001b, Scientific Impact of Large Telescopes, ING Newsletter, 4, 15

Crabtree, D. R. \& Bryson, E. P., 2003, Observatory Publications and Citations, Library and Information Services in Astronomy IV (LISA IV), Emerging and Preserving: Providing Astronomical Information in the Digital Age, Eds. Brenda G. Corbin, Elizabeth P. Bryson, and Marek Wolf (Washington, DC: U. S. Naval Observatory), 199

Crabtree, D., 2008, Scientific Productivity and Impact of Large Telescopes, SPIE, 7016, 70161A

Davoust, E. \& Schmadel, L. D., 1987, A Study of the Publishing Activity of Astronomers Since 1969, PASP, 99, 700

Dietrich, J. P., 2008, The Importance of Being First: Position Dependent Citation Rates on arXiv:astro-ph, PASP, 120, 224

Frogel, J. A., 2010, Astronomy's Greatest Hits: The 100 Most Cited Papers in Each Year of the First Decade of the 21st Century (2000-2009), PASP, 122,1214

Gal-Yam, A., et al., 2009, SN 2007bi as a PairInstability Explosion, Nature, 462, 624
Gaudi, B. S., et al., 2008, Discovery of a Jupiter/Saturn Analog with Gravitational Microlensing, Science, 319, 927

Grothkopf, U., Leibundgut, B., Macchetto, D., Madrid, J. P., \& Leitherer, C., 2005, Comparison of Science Metrics Among Observatories, The Messenger, 119, 45

Kamphuis, P. \& van der Kruit, P. C., 2010, Citations and Impact of Dutch Astronomy, arXiv:1011.5311

Kim, S. C., 2011, Paper Productivity of GroundBased Large Optical Telescopes from 2000 to 2009, PASA, 28, 249

Krause, O., et al., 2008, Tycho Brahe's 1572 SN as a Standard Type Ia as Revealed by Its Light-Echo Spectrum, Nature, 456, 617

Lee, J.-W., Kang, Y.-W., Lee, J., \& Lee, Y.-W., 2009, Enrichment by Supernovae in Globular Clusters with Multiple Populations, Nature, 462, 480

Lee, M. G., Park, H. S., \& Hwang, H. S., 2010, Detection of a Large-Scale Structure of Intracluster Globular Clusters in the Virgo Cluster, Science, 328,334

Leverington, D., 1996, The Cost-Effectiveness of Observational Astronomical Facilities Since 1958 Part I: Effectiveness, Q. J. R. astr. Soc., 37, 643 Leverington, D., 1997, Optical Telescopes - Biggest Best?, Nature, 385, 196

Meylan, G., Madrid, J. P., \& Macchetto, D., 2004, Hubble Space Telescope Science Metrics, PASP, 116,790

Pearce, F., 2004, Citation Measures: Citation Measures and Impact within Astronomy, Astronomy \& Geophysics, 45b, 15

Pimbblet, K. A., 2011, The h-index in Australian Astronomy, PASA, 28, 140

Ringwald, F. A., Culver, J. M., Lovell, R. L., Kays, S. A., \& Torres, Y. V., 2003, The Research Productivity of Small Telescopes and Space Telescopes, BAAS, 35, 1063

Ryu, D., Kang, H., Cho, J., \& Das, S., 2008, Turbulence and Magnetic Fields in the Large-Scale Structure of the Universe, Science, 320, 909

Sánchez, S. F. \& Benn, C. R., 2004, Impact of Astronomical Research from Different Countries, Astron. Nachr., 325, 445

Schawinski, K., et al., 2006, Suppression of Star Formation in Early-Type Galaxies by Feedback from Supermassive Black Holes, Nature, 442, 888 
Schawinski, K., et al., 2008, Supernova Shock Breakout from a Red Supergiant, Science, 321, 223

Schulman, E., French, J. C., Powell, A. L., Eichhorn, G., Kurtz, M. J., \& Murray, S. S., 1997, Trends in Astronomical Publication between 1975 and 1996, PASP, 109, 1278

Stanek, K. Z., 2008, How Long Should an Astronomical Paper Be to Increase Its Impact?, arXiv:0809.0692

Stanek, K. Z., 2009, Are Astronomical Papers with More Authors Cited More?, arXiv:0912.1855

Trimble, V., 1995, Papers and Citations Resulting from Data Collected at Large, American Optical Telescopes, PASP, 107, 977

Trimble, V., 1996, Productivity and Impact of Large Optical Telescopes, Scientometrics, 36, 237

Trimble, V., 2009, Telescopes in the Mirror of Scientometrics, Exp. Astron., 26, 133

Trimble, V., Zaich, P., \& Bosler, T., 2005, Productivity and Impact of Optical Telescopes, PASP, 117, 111

Trimble, V. \& Ceja, J. A., 2007, Productivity and Impact of Astronomical Facilities: A Statistical Study of Publications and Citations, Astron. Nachr., 328, 983

Trimble, V. \& Ceja, J. A., 2008, Productivity and Impact of Astronomical Facilities: Three Years of Publications and Citation Rates, Astron. Nachr., 329, 632

Yoon, S.-J., Yi, S. K., \& Lee, Y.-W., 2006, Explaining the Color Distributions of Globular Cluster Systems in Elliptical Galaxies, Science, 311, 1129 